\title{
Assessing the Impact of Demand Flexibility on Distribution Network Operation
}

\author{
B. D. Tavares, J. Sumaili, F. J. Soares, A. G. Madureira and R. Ferreira \\ INESC TEC, Porto, Portugal
}

bruna.c.tavares@inesctec.pt, jean.sumaili@inesctec.pt, filipe.j.soares@inesctec.pt, andre.g.madureira@inesctec.pt, rjcf@inescporto.pt

\begin{abstract}
This paper presents a study about the influence of Distributed Energy Resources' (DER) flexibility on the operation of a Medium Voltage (MV) network, in a Smart Grid (SG) environment. An AC multi-temporal Optimal Power Flow (OPF) tool was developed and used to simulate the impact of the DER flexibility (including storage devices, EVs, controllable loads and micro-generation) in distribution network operation. Some simulations are presented, demonstrating the impact that DER flexibility can have on solving operation problems namely in terms of branch loading and voltage limits.
\end{abstract}

Index Terms - Distributed energy resources, Load shifting, Multi-temporal OPF, Smart grids.

\section{INTRODUCTION}

A new paradigm in power system has been the theme of discussion due to the increasing integration of distributed energy resources (DER) [1]. To take full advantage of the DER presence in power systems, network observability and controllability need to be increased, which can be more easily achieved through the implementation of smart grid (SG) technologies and functionalities.

This new paradigm increases the complexity of network operation process and creates new and interesting challenges that should be further investigated such as the optimal management of resources' flexibility and their utilization in enhancing network operation. The integration of DER in distribution networks is already known to bring some problems related to voltage and branch limits violations, principally in the peak load periods. However, the integration of such resources also increases the network's overall flexibility.

Several studies about the DER alternatives and impacts on the network operation have been conducted. Regarding storage devices (SD), in addition to analysis of the impacts on the network, there are comparison between diverse technologies [2], different formulations to determine the optimal operation depending on the entity owning the resources [3] and even studies about the aggregation between SD and distributed generation (DG) [4]. For electric vehicles (EV), not only exists frameworks to successfully integrate electric vehicles into electric power systems [5], but also studies about the potential economic implications of using vehicle batteries to grid storage devices [6]. In overall, these studies show the need to develop new control and management architectures as presented in [1].

This paper takes a step beyond previous works by focusing on the optimal management of the different types of flexibility that DER can provide, such as the capability of load shifting (including EV), operation of SD in low voltage (LV) networks, and renewable energy production. As the proposed methodology focuses on medium voltage (MV) networks, some simplifications were made for LV grids: they are assumed to have unlimited capacity to integrate DER.

The evaluation of the DER flexibility impact on the MV grid operation was performed using a multi-temporal Optimal Power Flow (OPF), described in section II. The algorithm, adapted from [4], was developed using an approach to minimize the distribution network operation cost, taking advantage of the DER flexibility, and maximize at the same time the integration of renewable energy. The implemented method seeks for an optimal solution through the resource utilization resulting on a lower cost over the selected periods, in a way that voltage and branches limits are always respected. For that, the network duplication is computed through different periods of time taking into consideration inter-temporal constraints.

\section{PROBLEM FORMULATION}

The multi-temporal OPF tool was developed using an OPF based on the MatPower OPF formulation [7], where not only some OPF constraints, but also the objective function, are already implemented. The description of the objective function and problem's constraints is provided in the following section. Some additional constraints, related to the DER usage, are also described.

\section{A. Objective function}

The optimization vector, $\mathrm{x}$, for the AC multi-temporal OPF consists of voltage angles $(\theta)$, voltage magnitudes $\left(V_{m}\right)$, and active and reactive generated power $\left(P^{g}\right.$ and $Q^{g}$, respectively). The objective function is simply a summation of individual polynomial cost functions of real and reactive power injections $\left(f^{P}\right.$ and $\left.f^{Q}\right)$ and is given by :

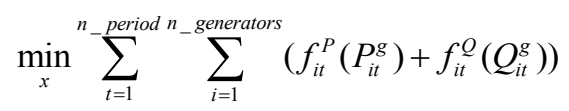

This work is financed by the FCT - Fundação para a Ciência e a Tecnologia (Portuguese Foundation for Science and Technology) within project MITPTB/CS/0026/2013 and project SmartGP/0002/2015 under the framework of ERA-Net Smart Grids Plus. 
where $f_{i t}$ is the cost function of the generator $i$ in time period $t$ and $P_{i t}^{g}$ is the production from the generator $i$ in the respective time period.

\section{B. Problem constraints}

In addition to the classic OPF constraints, (2) to (4), some others are considered, either related to inter-temporal dependencies (e.g. the state of charge of batteries) or related to load shifting.

$$
\begin{aligned}
& \sum_{k=1}^{n_{-} \text {gen }{ }^{i n}{ }^{i}} P_{i k}^{g}-P_{i}^{\text {load }}=\sum_{j=1}^{N} V_{i} V_{j}\left[G_{i j} \cos \theta_{i j}+B_{i j} \sin \theta_{i j}\right] \\
& \sum_{k=1}^{n_{-} \text {gen }{ }^{i n_{-} i}} Q_{i k}^{g}-Q_{i}^{\text {load }}=\sum_{j=1}^{N} V_{i} V_{j}\left[G_{i j} \sin \theta_{i j}-B_{i j} \cos \theta_{i j}\right] \\
& S_{i j}^{\min } \leq S_{i j} \leq S_{i j}^{\max } \\
& x_{i}^{\min } \leq x_{i} \leq x_{i}^{\max } \\
& S o C_{s t}^{\min } \leq S o C_{s t} \leq S o C_{s t}^{\max } \\
& \tan _{l t}(\varphi) * P_{l t}^{g}-Q_{l t}^{g}=0 \\
& S o C_{e T}=p^{*} S o C_{e}^{\max } \\
& \sum_{t=1}^{n} \text { periods }\left(P_{l t}^{\text {increased }}-P_{l t}^{\text {decreased }}\right)=0
\end{aligned}
$$

where:

- Equations (2) to (4) are the traditional OPF equality and inequality constraints. (2) and (3) represents the power balance equations and (4) symbolizes the set of equations corresponding to branch flow limits;

- Equation (5) represents the problem variables limits, such as voltage angle and magnitudes and generators active and reactive power generation limits;

- $S o C_{s}$ - State of Charge of the device $s$, where $s$ represents the storage elements, including $\mathrm{EV}$ batteries. The SoC is bounded by a minimum and maximum value, guaranteed by (6) ;

- $\tan _{l t}(\varphi)$ - Relation between the active and reactive power of the original load. (7) assures that $P_{l t}^{g}$ and $Q_{l t}^{g}$ in the variable $l$, which represent the load increase or decrease, at time $t$, maintain the same proportion as the original load;

- The constraint (8) assures that the EV batteries' SoC is at a certain percentage, $p$, of its maximum capacity at a specific time (e.g., the time of disconnection). In this study, $p$ was set as $100 \%$;

- (9) Assures that the entire decreased load is replaced in some other period.

\section{Model assumptions}

Some assumptions and simplifications were made in the model, as described in the next paragraphs.

The network used as a test case was a MV network where each Secondary Substation (SS) has a simplified representation of the downstream network: the data related to the flexible DER in the LV network is aggregated and jointly represented in the SS. Thus, each SS has associated seven variables, which represent: the power consumption of the domestic SD; the power injected by the domestic SD; the power consumption of the $\mathrm{EV}$; the power injected in the network by the EV; the potential load decrease of flexible loads; the potential load increase of flexible loads; and the photovoltaic (PV) production.

The domestic storage devices' consumption/injection profiles are highly influenced by the market price, or tariffs, and auto-production. Thus, aiming at a more realistic profile, these resources are optimized (by the end user perspective electricity cost minimization) in a first stage and then used as input of the multi-temporal OPF.

The optimal domestic SD operation was defined based on the formulation presented in II B, but considering only the constraints (2), (3), (6), (8) and (9), like the formulation proposed in [3]. The domestic SD profile previously computed is introduced in the main problem and the cost considered for that profile is zero. If the optimal profile is changed by the multi-temporal OPF, the cost increases proportionally to the deviation.

As referred, each SD (batteries and EV) is represented by two variables: $P^{\text {cons }}$ and $P^{i n j}$. The SoC of device $s$ at time $t$ is represented by :

$$
S o C_{s t}=\sum_{j=1}^{t}\left(-\varepsilon_{s}^{c o n s} * P_{s t}^{c o n s}-\frac{1}{\varepsilon_{s}^{i n j}} * P_{s t}^{i n j}\right)
$$

where $\varepsilon^{\text {cons }}$ and $\varepsilon^{i n j}$ are respectively the devices' charging and discharging efficiencies.

A parameter referring to the percentage of load flexibility is also included. The load can be adjusted as it is more convenient inside these limits, but with a higher cost. Outside these limits it is not possible to change the load levels. The power factor in the remaining load (after the adjustment) is assumed to remain with the same initial value. So, the power factor of the decrease/increase load is forced to assume that same value. This constraint is indirectly obtained by (7), where the relation between $\mathrm{P}^{\mathrm{g}}$ and $\mathrm{Q}^{\mathrm{g}}, \tan (\varphi)$, is computed.

Regarding EV, it is assumed that they are connected to the network during the following periods: 0 to $7 \mathrm{~h}$ and 20 to $24 \mathrm{~h}$. It is also assumed that every EV takes at least $4 \mathrm{~h}$ to fully recharge its batteries. The number of EV in each SS is assumed to be proportional to the load. The DSO can freely operate the $\mathrm{EV}$ providing that the restriction represented in (8) is respected.

Distributed renewable energy sources flexibility was also considered. Their production can be decreased (from a forecasted profile) according to a defined flexibility level, but a higher cost will be associated.

\section{PRELIMINARY RESULTS AND DISCUSSION}

\section{A. Case studies}

A network based on a real $15 \mathrm{kV}$ Portuguese network was used to test the proposed model. In order to access the DER flexibility impact in the network operation, five different scenarios were compared in an hourly basis. 
Scenario 1 corresponds to the network with its original conditions (base case), without PV production or EV, operating during a day with typical domestic load profiles, with different power demand from each SS.

Scenario 2, includes PV production and EV in each SS, but no DER flexibility is considered. The percentage of EV power relating to the maximum load demand in the $\mathrm{SS}$ is $40 \%$. PV has a maximum production corresponding to $70 \%$ of the residential load demand.

Scenario 3 assumes flexibility on the EV charging period, where the EV have the possibility to charge during a certain period, as opposed to what happens in the second scenario (EV charge continuously right after being plugged-in to the grid, at 7 p.m.). In the present scenario, EV charging has the possibility to be managed between 7 p.m. and 7 a.m.

Scenario 4 considers that also domestic loads have flexibility. It was assumed the possibility of load shifting by moving the consumption to any other period of the day. The load flexibility was defined as $15 \%$ of the domestic load.

Scenario 5 does not consider domestic loads flexibility, but includes domestic SD in the LV network, where the installed power corresponds to $20 \%$ of the residential load demand. This scenario includes two steps. In the first step, the optimal profile for all the SD is calculated from the end user perspective (i.e. with the objective of maximizing end users' profit). Such profile is calculated based on the tri-hour tariffs established for end users in Portugal [8]. The second step is related to the utilization of the multi-temporal OPF to minimize grid operational costs and avoid network constraints violations. This step establishes the deviation from the profile computed in the first step. This deviation is regarded as a flexibility service provided by end users to the DSO and uses the market price as input, such as in the previous scenarios.

\section{B. Results comparison}

The network in its initial operating conditions (Scenario 1) has branches loading far from the limits and acceptable voltage levels. Fig. 1 presents the network voltage profile for this first scenario.

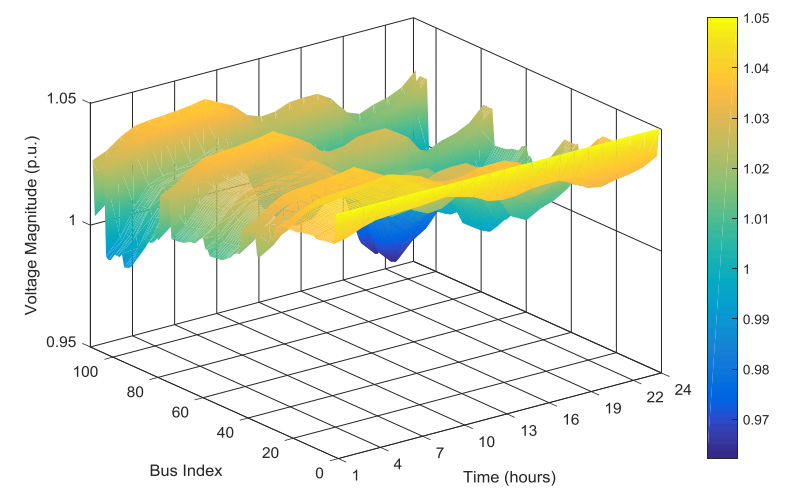

Figure 1. Network voltage profile for Scenario 1.

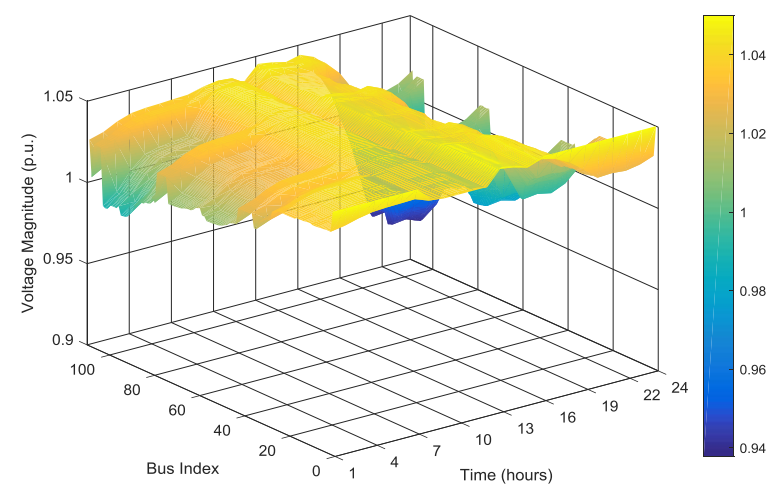

Figure 2. Network voltage profile for Scenario 2.

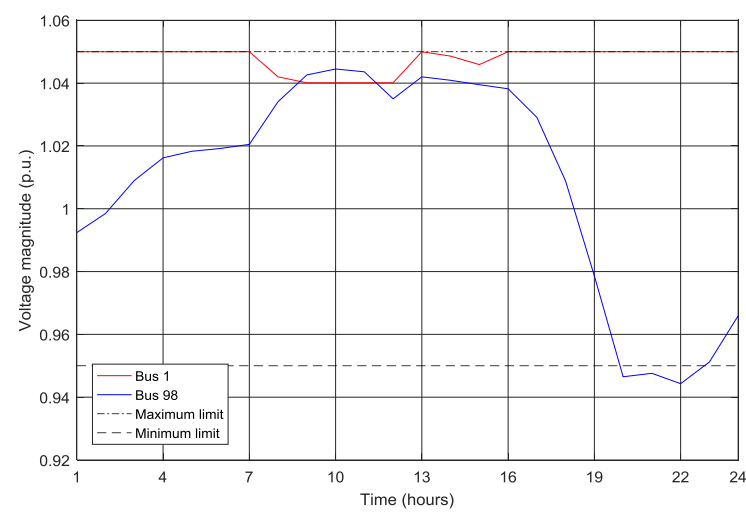

Figure 3. Voltage profile (Scenario 2) in two buses, slack (bus 1) and one with extreme voltage behavior (bus 98).

In Scenario 2, with the integration of PV and EV, the network voltage profile undergoes significant changes, as shown in Fig. 2 and Fig. 3.

First, the peak of consumption around 8 p.m. increases because the EV consumption superimposes on the load peak. This additional consumption leads to violation of the specified voltage limits, [0.95; 1.05] p.u.. Second, around 11 a.m. the PV production exceeds the load consumption causing an inversion of the power flow in the primary substation, as shown in Fig. 8, which leads to a situation where the voltage in the primary substation (bus 1) is lower than in the other buses (Fig. 3). Assuming that power injection in the transmission network is not allowed, there are two options: either decrease the PV production or shift some load to that period of high renewable production.

Regarding Scenario 3, the EVs are usually connected to the grid on the peak hours, and stay connected during the entire night, until they are unplugged in the next day. As mentioned, the periods when EV are charged can be optimized in this scenario (between 7 p.m. and 7 a.m.). The impact on the voltage profile is visible in Fig. 4 and Fig. 5. 


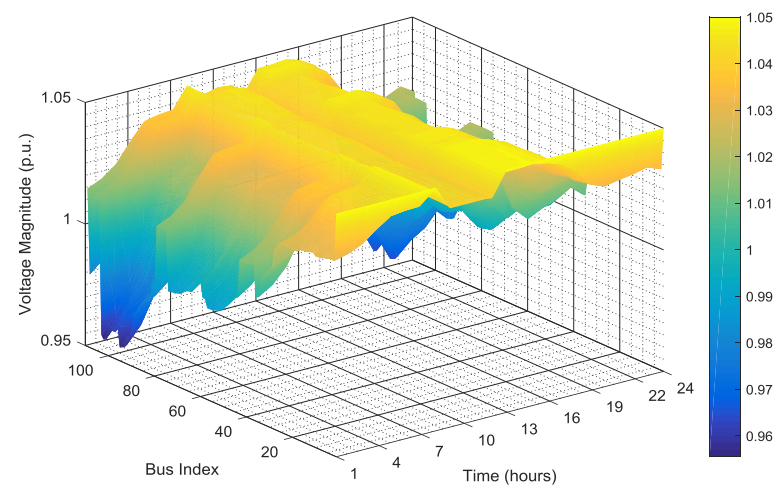

Figure 4. Network voltage profile for Scenario 3.

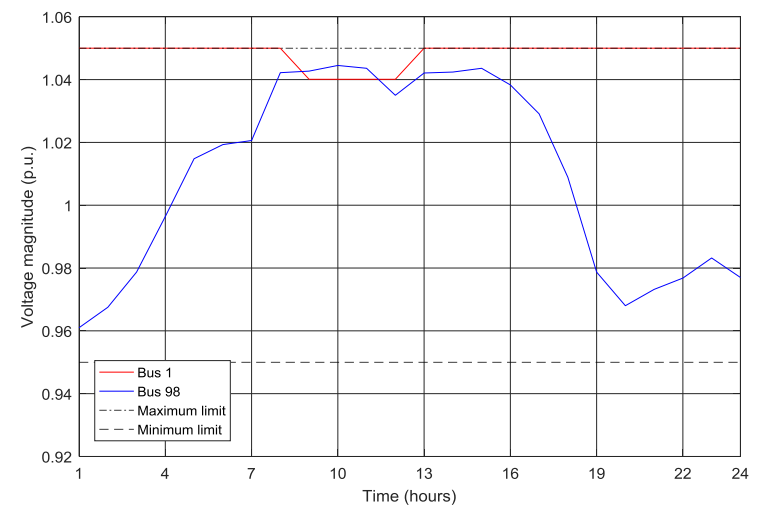

Figure 5. Voltage profile (Scenario 3) in two buses, slack (bus 1) and one with extreme voltage behavior (bus 98).

The voltage problems identified in Scenario 2 were solved just by moving the EV loading from the peak hours (7 p.m. to 11 p.m., Fig. 6) to the off-peak hours (11p.m. to 4a.m.), as shown in Fig. 7. This approach yields two benefits: the EV do not charge all at the same time and the charging is on the offpeak hours. Besides solving the voltage problems, it also decreases the total active power losses from 1.66 to 1.49 MW.

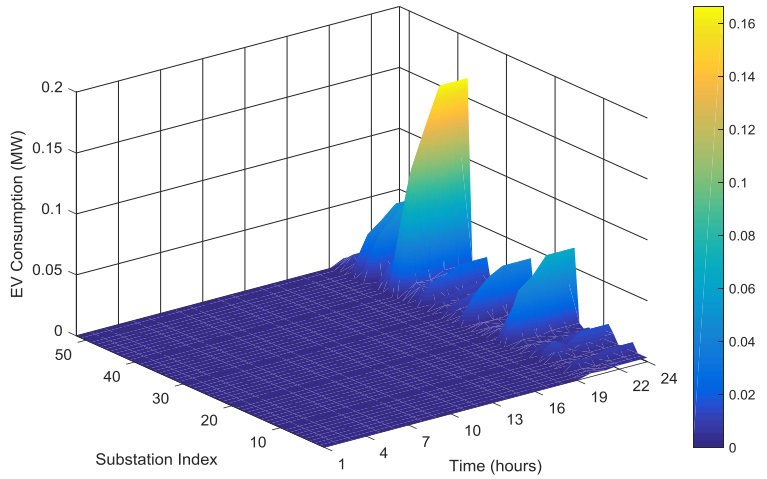

Figure 6. EV charging profile without charging flexibility - Scenario 2.

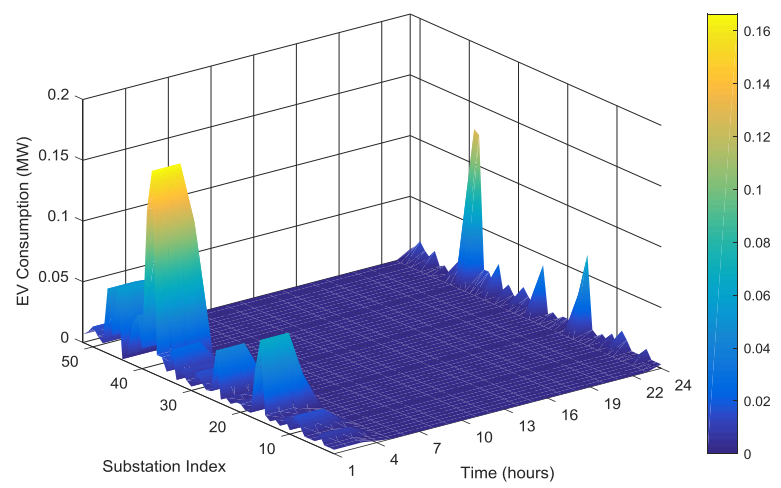

Figure 7. EV charging profile with charging flexibility - Scenario 3.

In Scenario 4, it is shown that load flexibility allows solving the problem of inverted power flows in the primary substation, as depicted in Fig. 8 . Actually, with only 15\% of load flexibility, the voltage in the primary substation is equal or higher than the other buses, as shown in Fig.9 and Fig.10.

In Fig.11 it is possible to observe the amount of load that was moved to the hours with the maximum PV production. In addition to the mentioned benefits, the load flexibility also contributes to reduce the active power losses from 1.66 to $1.40 \mathrm{MW}$.

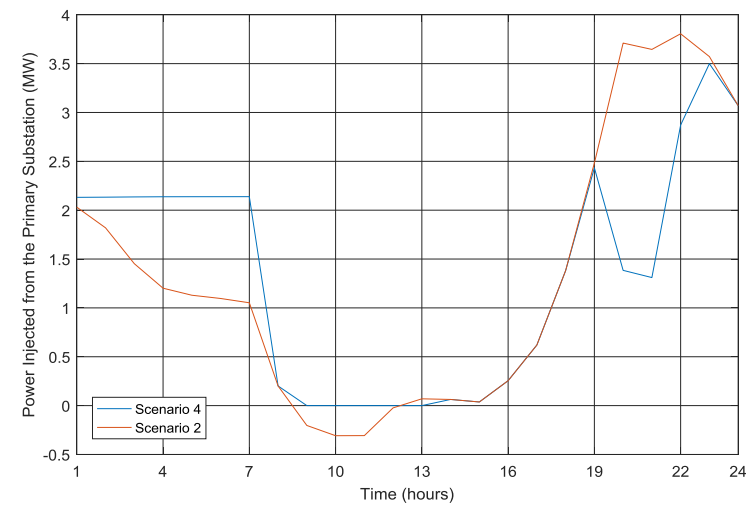

Figure 8. Power injected from the primary substation.

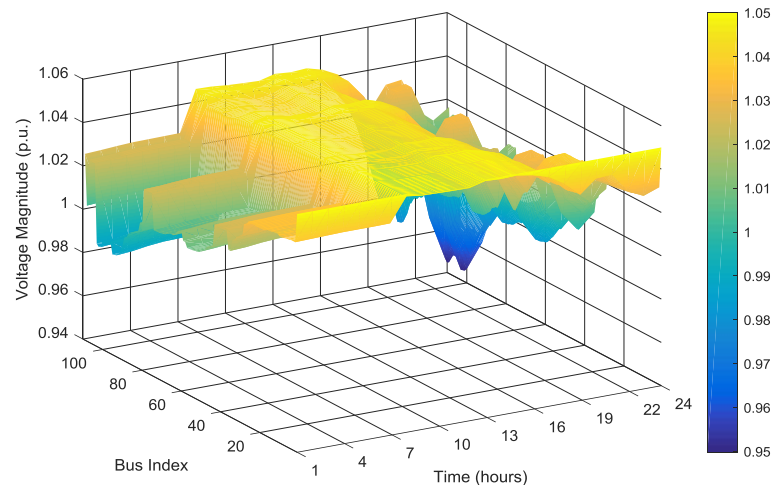

Figure 9. Network voltage profile for Scenario 4. 


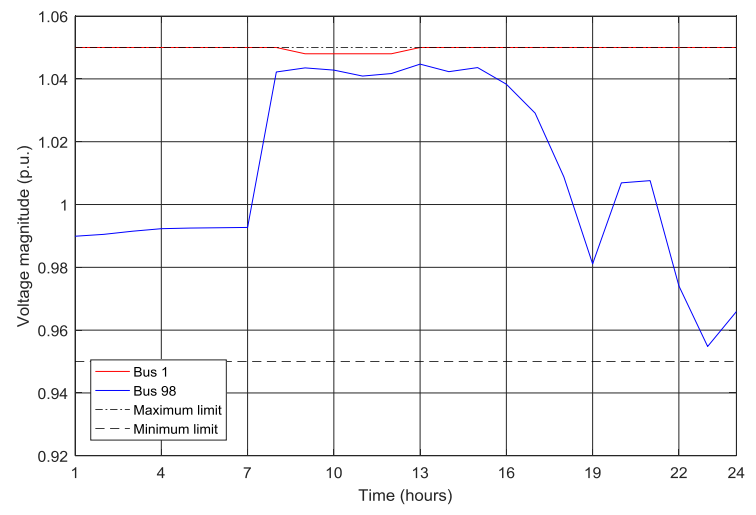

Figure 10. Voltage profile (Scenario 4) in two buses, slack (bus 1) and one with extreme voltage behavior (bus 98).

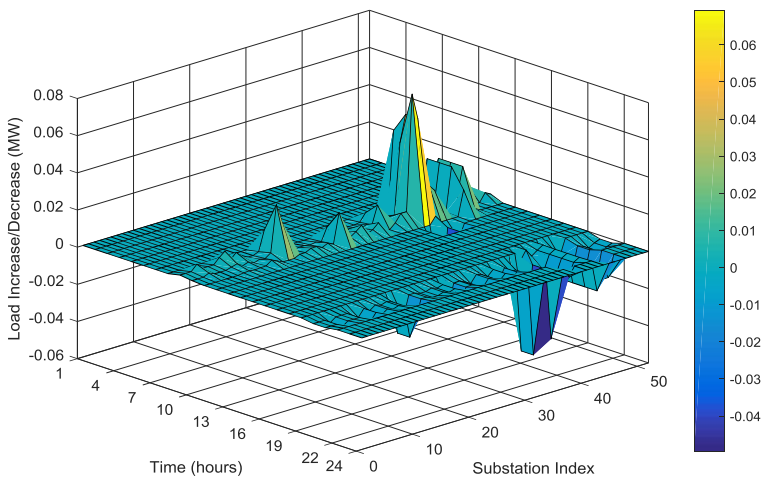

Figure 11. Load increase/decrease (MW) for Scenario 4.

The integration of DER and EV in distribution networks is already known to bring some problems related to voltage and branch limits violations and power flow inversions. Some of these problems where portrayed in Scenario 2. However, in Scenario 3 and 4 , it was demonstrated that the coordinated operation of such resources could easily mitigate the detected problems. The use of DER flexibility presents also some other benefits, such as increasing renewable energy sources integration, decreasing operation costs by shifting load from the peak hours to periods with high renewable production and improving the overall network operation, which contributes to decrease active power losses.

As previously referred, in Scenario 5 the domestic SD are first operated in a way that maximizes the clients' profit. The remuneration for power injected in the network by the clients has the value of $90 \%$ of the average market price in the previous month. In this first step, the energy prices are obtained from the tri-hour tariffs mentioned above, and they are used to optimize SD behavior according to real energy injection/consumption prices. In the second step, as it is an optimization made by the DSO, real market prices were used. Both the tri-hour tariffs and market prices are shown in Fig. 12 , together with the end user remuneration for the power injected and the assumed price for the flexibility service provided by end-users to the DSO.

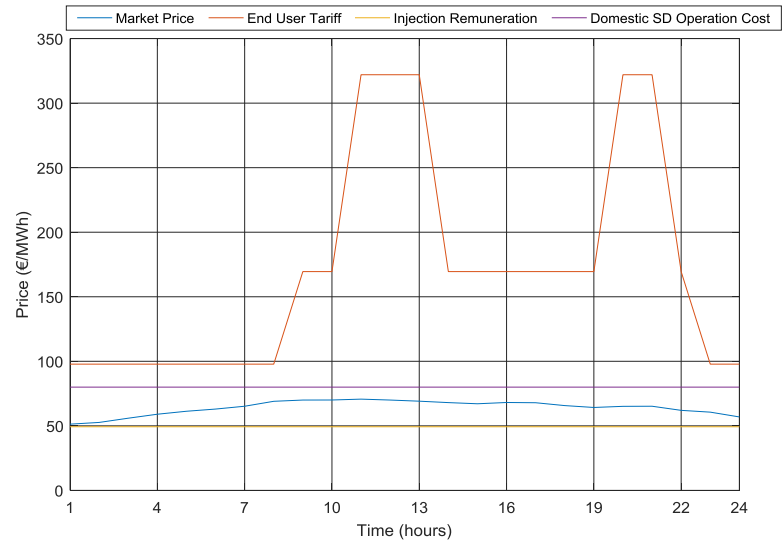

Figure 12. Tariffs, market prices, end user remuneration for injected power and proposed cost for the flexibility service provided by end users SD to the DSO (€/MWh).

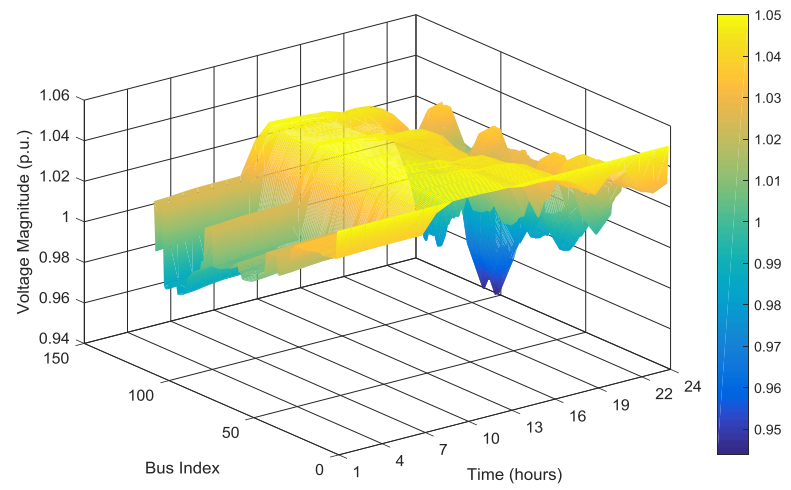

Figure 13. Network voltage profile for Scenario 5, considering domestic $\mathrm{SD}$ operated under the end user perspective.

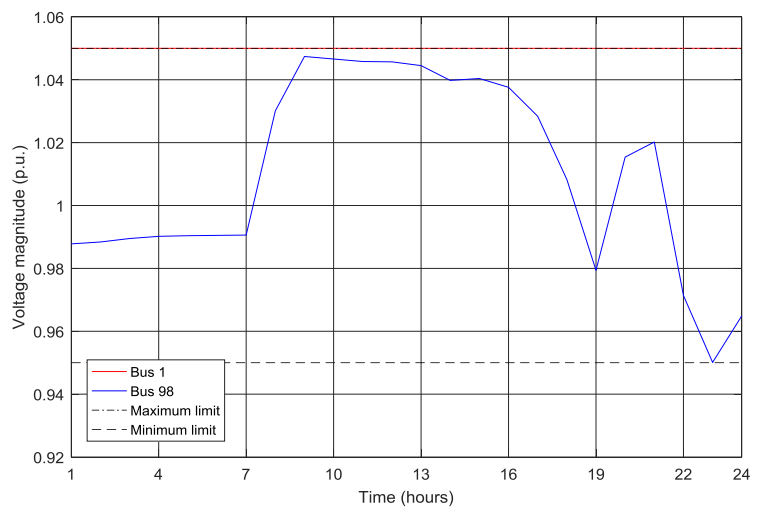

Figure 14. Voltage profile (Scenario 5) in two buses, slack (bus 1) and one with extreme voltage behavior (bus 98).

In Fig. 13 and Fig.14 is possible to conclude that the inclusion of domestic SD, operated only by the clients, eliminates the voltage violations. In fact, the SD behavior is highly influenced by the consumption needs and the tariffs, allowing to deviate the network consumption from in peak hours to valley hours, where tariffs consist on lower prices. Furthermore, SD allows to save produced power (from DG) for latter consumption. Thus, the combination between tariffs 
and SD allows decreasing the network power flows in the periods where voltage constraints were violated due to excessive power consumption.

It was assumed that DSO could operate the domestic SD, paying for the deviation from the previous drawn profile. In this case, the DSO does not have the need to operate the domestic SD to eliminate the voltage violations. However, it stablished a cost for the deviation of $80 € / \mathrm{MWh}$, which is near to the maximum market price, between 11 and $16 \mathrm{~h}$. Observing Fig. 15 and Fig. 16 it is easy to conclude that for these hours is more beneficial to use the SD flexibility than the power from the upstream network, avoiding network losses. Nevertheless, as the deviation cost increases, both domestic SD profiles get closer, since there are no violated constraints.

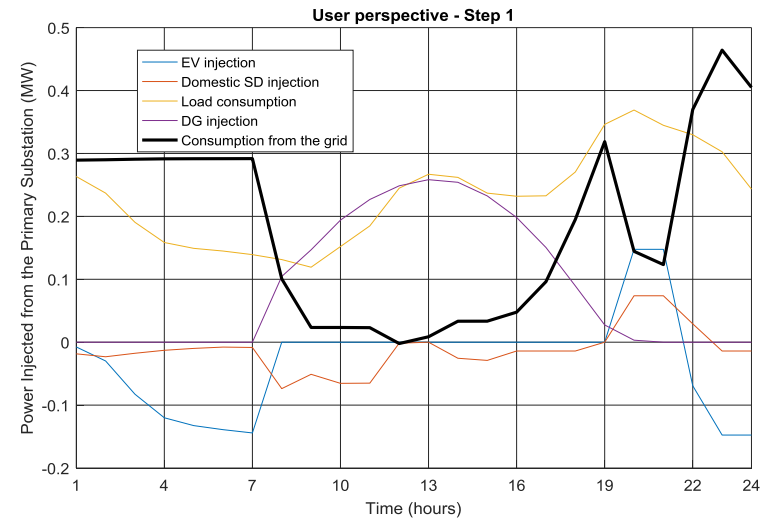

Figure 15. Consumption/injection profiles in bus 89 . Profiles calculated considering the end user perspective.

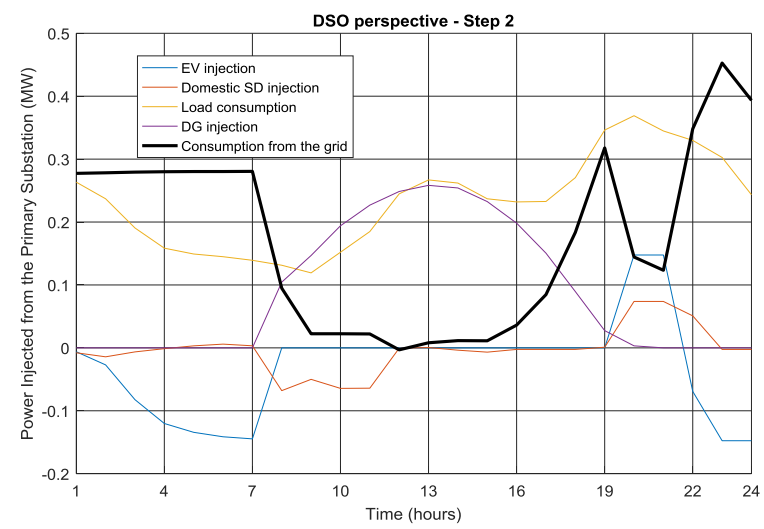

Figure 16. Consumption/injection profiles in bus 89 . Profiles calculated considering the DSO perspective.

\section{CONCLUSIONS}

In this paper was possible to observe some of the problems DER integration can bring to the network operation, namely voltage violations, during peak hours, and reversed power flows, during high production hours. Although DER integration bring such problems, it also increases the network overall flexibility. If one aims at maximizing the DER integration, the way to deal with these problems may be by exploiting the DER flexibility. As shown in section III, DER flexibility can deal with voltage problems and improve the overall grid operation performance.

Flexibility in the EV charging periods may help attenuating the increased demand peak due to EV consumption during the peak hours. Load flexibility shows the advantage of enabling load shifting from load peak periods to periods with high DG availability, decreasing the network losses and avoiding reverse power flows. It also has the advantage of solving problems related to voltage limits. Although there are no branches loading problems on the analyzed cases, it is understandable that such problems would be easily solved applying the same approach used for the presented voltage violation cases, either by load sifting (including not only residential load, but optimization of EV and SD charging profiles) or the excessive production curtailment.

The end user tariffs are also a powerful tool to smooth the total consumption profile, influencing the end user behavior. It has a major influence especially if the number of SD in the network is very large.

The results achieved show that the exploitation of DER flexibility may be a very interesting alternative for system operators. When compared with traditional grid reinforcement investments, DER flexibility will be rather inexpensive and easy to implement in smart grid environments, where the necessary communication infrastructure will be already available together with the smart metering system.

\section{REFERENCES}

[1] A .Madureira, C. Gouveia, C. Moreira, L. Seca, J. Peças Lopes, "Coordinated management of distributed energy resources in electrical distribution systems", in iEEE PES innovative Smart GridTechnologies Latin-America (ISGT LA), Sao Paulo, 2013, pp. 1-8.

[2] Bradford P. Roberts and Chet Sandberg. "The role of energy storage in development of smart grids." Proceedings of the IEEE 99.6 (2011): 1139-1144.

[3] Ricardo Ferreira; Manuel Matos; João P. Lopes. "Regulatory issues in the deployment of distributed storage devices in distribution networks". In: European Energy Market (EEM), 2016 13th International Conference on the. IEEE, 2016. p. 1-6.

[4] Souza, S. M., M. Gil, J. Sumaili, A. G. Madureira and J. A. Peças Lopes. "Operation scheduling of prosumer with renewable energy sources and storage devices". In: European Energy Market (EEM), 2016 13th International Conference on the. IEEE, 2016. p. 1-5.

[5] João A. Peças Lopes, Filipe Joel Soares, and Pedro M. Rocha Almeida. "Integration of electric vehicles in the electric power system." Proceedings of the IEEE 99.1 (2011): 168-183.

[6] Peterson, Scott B., J. F. Whitacre, and Jay Apt. "The economics of using plug-in hybrid electric vehicle battery packs for grid storage." Journal of Power Sources 195.8 (2010): 2377-2384.

[7] Ray D. Zimmerman, Carlos E. Murillo-Sánchez. MatPower Tool available online at: http://www.pserc.cornell.edu/matpower

[8] ERSE. Regulated Tariffs and prices for 2015. Available at: http://www.erse.pt/ 